\title{
Flame propagation and acceleration in narrow channels: sensitivity to facility specific parameters
}

\author{
Yves Ballossier • Florent Virot • \\ Josué Melguizo-Gavilanes
}

Received: date / Accepted: date

\begin{abstract}
Flame propagation experiments in stoichiometric $\mathrm{H}_{2}$-air are conducted in a smooth 482-mm long, $10-\mathrm{mm} \times 10$-mm square cross section channel, closed at the ignition end and open at the opposite end. Direct observation is used to track the flame acceleration dynamics. The effect of facility specific parameters (i.e. ignition energy, boundary conditions near the ignition end, window material and settling time between filling and ignition) on flame propagation and acceleration is assessed. The absolute mean deviation is used as a metric to determine the effect of the aforementioned parameters on the collected front position data, and Response Surface Methods for experimental design is applied to examine inter-parameter interactions. Results show that the boundary conditions near the ignition end (i.e. large/small ignition offset) have the largest influence on the recorded front position with the remaining parameters playing a less important role. The early stages of flame propagation is compared against theoretical predictions to understand the discrepancies observed in the data; a simple acoustic model was found sufficient to explain the increased acceleration rates observed for the large ignition offset cases. Finally, a link between the observed front oscillations during flame acceleration and the flow rate dynamics at the channel's open end is highlighted.
\end{abstract}

Keywords flame acceleration · narrow channels · hydrogen · experimental characterization

Yves Ballossier

Institut Pprime, UPR 3346 CNRS, ISAE-ENSMA

BP 40109, 86961, Futuroscope Chasseneuil Cedex, France

Tel.: +33(0)549498080

Fax: +33 (0)549498000

E-mail: yves.ballossier@ensma.fr 


\section{Introduction}

From a scientific point of view, deflagration-to-detonation transition (DDT) continues to draw significant interest in the research community as an outstanding, physics-rich fundamental problem in combustion science. From a practical perspective, it is important to study and understand DDT in order to develop engineering correlations and simulation tools that can be applied to the prevention and mitigation of explosions [1], [2], as well as to novel propulsion applications [3].

The one-dimensional flow structure upon abrupt ignition of a reactive mixture at rest is composed of a shock, and a trailing temperature interface, i.e., a flame. Depending on the boundary conditions (close vs. open at the ignition end) the expansion of burned products will play a greater or lesser role in the early flame dynamics. In open geometries, flames accelerate due to their intrinsic instabilities and interactions with the ignition-induced flow, increasing its surface area. In confined geometries, on the other hand, specifically within channels or tubes, pressure builds up providing an additional acceleration mechanism for the flame. Mixtures with large expansion ratios result in higher acceleration rates and earlier development of precursor shocks during initial flame propagation and subsequent acceleration [4].

In obstructed channels with large cross-sections $(\mathcal{O}(\mathrm{cm})$ to $\mathcal{O}(\mathrm{m}))$ it is relatively well established that transition to detonation occurs upon multiple reflections of the precursor shocks that form ahead of the flame during the acceleration stage. In unobstructed channels with small cross-sections $(\mathcal{O}(\mathrm{mm}))$, successive precursor shocks heat up the reactive mixture, providing favorable feedback for flame acceleration until detonation onset is possible. The exact DDT mechanisms for the latter length scale require further study as boundary layer effects may play an important role in every stage of the DDT process [5][8]. High quality experimental data collected in narrow channels is thus needed to characterize flame acceleration (FA) and to the overall understanding of DDT.

To that end, here, we present a $482-\mathrm{mm}$ long channel with a $10-\mathrm{mm} \times$ $10-\mathrm{mm}$ cross section, closed at the ignition end and open at the other end in which various design parameters are systematically analyzed. This is done to ensure repeatable results that avoid facility specific effects which may not be representative of the actual physics of FA. Careful understanding of how FA is affected by design parameters as well as by the chosen experimental methodology is a necessary step that will aid in the analysis of experimental DDT results as well as in future comparison with numerical simulations. Furthermore, the findings presented here will expedite the conception of new facilities by interested research groups, information we find to be lacking in the open literature.

In the present manuscript, we aim to answer the following questions: (i) for ignition energy levels significantly below those required for direct detonation initiation, how does the initial energy deposition affect FA; (ii) expansion of burned products has been identified as the main FA mechanism during the 
early stages of acceleration after ignition, and flames are known to be strongly affected by the boundary condition behind them (i.e. closed vs. open ends) [9], how does the relative position of the ignition electrodes with respect to the close end (referred to as ignition offset herein after) affect FA; (iii) does the settling time between the end of the filling process and ignition influence FA, from what point on can we assume quiescent flow prior to ignition that does not affect FA; and (iv) does the material utilized for optical access affect the recorded flame position.

To do so, the parameters listed above are studied for $\mathrm{H}_{2}$-Air in which the flame position as a function of time is recorded using direct observation. The global mean deviation is used as a metric to determine the effect of each parameter on the data collected, and Response Surface Methods for experimental design is applied to asses inter-parameter interactions. Finally, to further understand our data, the early-stages of flame propagation are analyzed using previous theoretical developments/simple acoustic models, and the flow-rate dynamics at the end of the channel as well as its relationship with the oscillations observed during FA are studied using schlieren visualization.

The manuscript is organized as follows: in section 2 the experimental setup, visualization and post-processing methodology are described. Sample front position data, and associated deviations are presented in section 3, focusing on ignition energy and the ignition offset to illustrate the methodology. In section 4, a thorough discussion is given, followed by concluding remarks in section 5. Finally, the remaining data collected during our parametric study, as well as additional details on the mathematical framework used to assess inter-parameter interactions, are included in Appendices 1, 2 and 3.

\section{Experimental setup, visualization and post-processing methodology}

2.1 The narrow channel and experimental procedure

A $10-\mathrm{mm} \times 10-\mathrm{mm}$ square cross-section, close-/open-end channel was machined on an aluminum block, and a sheet of polycarbonate (plastic) or glass was bolted as the fourth side of the channel to allow optical access for direct photography; see Fig. 1 - top. The mixture is ignited using electrodes, with a separation distance of 1-mm, located 14-mm from the close-end. The effective channel length from the ignition point to the open end is $482 \mathrm{~mm}$ of which $400 \mathrm{~mm}$ are optically accessible.

For each experiment, the channel is evacuated to an absolute pressure below $0.1 \mathrm{kPa}$ (measured by a MKS 220DA pressure sensor). A plastic cap, held by a servo motor linkage, seals the open end of the channel. Subsequently, the $\mathrm{H}_{2}{ }^{-}$ $\mathrm{O}_{2}-\mathrm{N}_{2}$ mixture, prepared in an external tank $(50 \mathrm{~L}$ bottle) using the method of partial pressures, is fed into the channel until atmospheric pressure is reached. At this point, the plastic cap is removed by the servo motor, and one second later the mixture is ignited at the closed end by the electric discharges that 


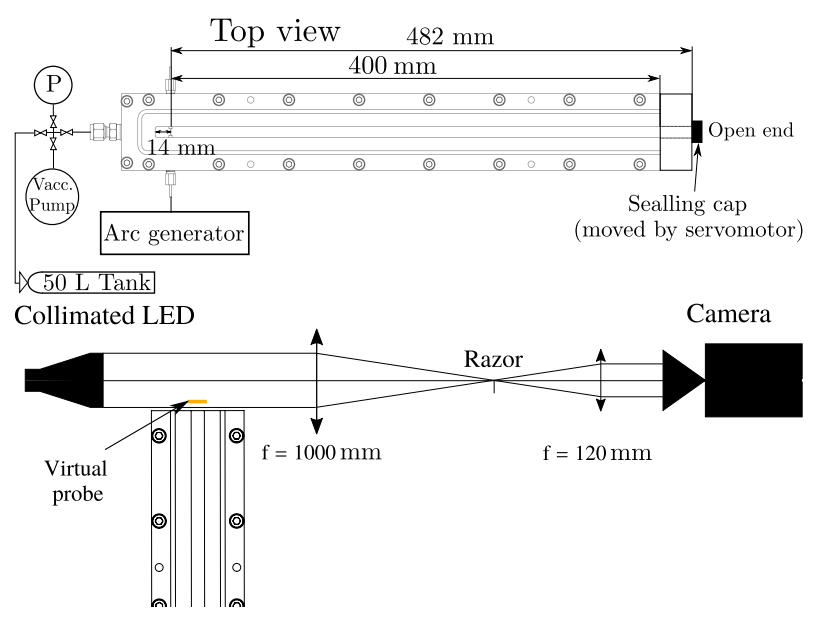

Fig. 1 Schematic of experimental setup (top) and schlieren visualization system at channel exit (bottom).

forms between the electrodes. After each test, the channel is evacuated for $\sim 5$ minutes to remove condensation from burned products.

\subsection{Flow Visualization}

The ignition and subsequent flame propagation inside the channel was visualized directly using a black and white Photron FASTCAM SA-Z equipped with a Sigma 17-50 mm lens. We captured the entire optical access of the channel $(400 \mathrm{~mm})$ at a spatial resolution of $1024 \times 24$ pixels $(\sim 0.45 \mathrm{~mm} / \mathrm{pixel})$. Stoichiometric $\mathrm{H}_{2}$-air has a laminar burning speed, $S_{L}=2.25 \mathrm{~m} / \mathrm{s}$, which correspond to a flame velocity, $u_{f}=\Theta S_{L}=15.42 \mathrm{~m} / \mathrm{s}$, where $\Theta$ is the expansion ratio of the mixture. A camera framing rate of $10,000 \mathrm{fps}$ is sufficient to capture the initial flame propagation and subsequent acceleration with a maximum exposure time of $99 \mu \mathrm{s}$. The same trigger was used for the camera and electric discharge generator to synchronize the data acquisition. Schlieren visualization was used at the channel exit to investigate the flame oscillatory behavior observed in the data collected; see subsection 4.3 for more details. An in-line configuration with the camera described above was used for that purpose. The setup included a 100-mm collimated white LED, and two BK7 lenses of 150-mm and 51-mm diameter with focal lengths of $1000 \mathrm{~mm}$ and 120 $\mathrm{mm}$, respectively. Cut-off was handled using a razor blade positioned at $45^{\circ}$ set to block about $50 \%$ of the light beam. Videos were recorded at 20,000 fps with an exposure of $347 \mathrm{~ns}$. The image resolution was set to $1024 \times 1024$ pixels. A schematic of the schlieren set up is shown in Fig.1 - bottom. 


\subsection{Front tracking}

The combustion front was automatically detected from the raw images using a post-processing script written in Python including the following steps: (i) the two-dimensional images are cropped to contain the line along the main front propagation direction where the luminous signal over the height of the channel is maximum; (ii) the peak noise value upstream of the front is then subtracted from the cropped images; (iii) the front is detected using a threshold set to a multiple of the maximum noise (i.e. between $0.5-1.5$ )

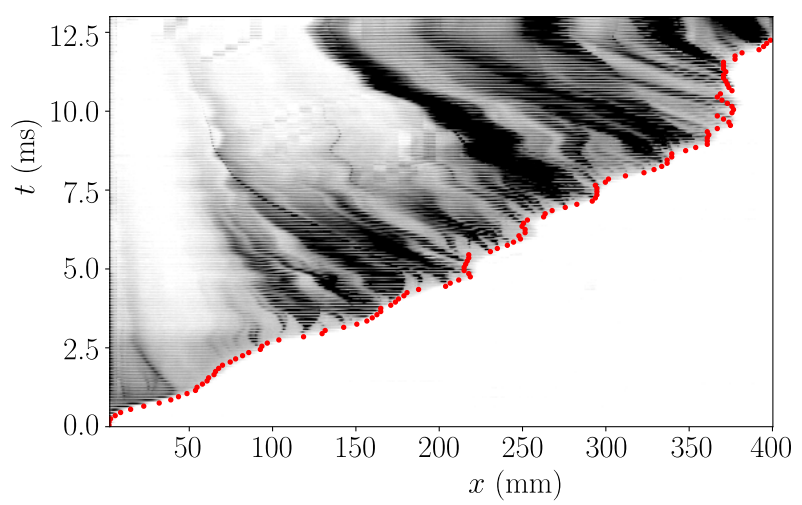

Fig. 2 Raw $x-t$ diagram (inverted colors) and result of front tracking. Configuration: Small ignition offset, plastic and Igniter 1; conditions: stoichiometric $\mathrm{H}_{2}$-air at $p_{0}=100 \mathrm{kPa}$, and $T_{0}=290 \mathrm{~K}$.

A sample raw image showing an $x-t$ diagram and associated luminous front detection (red points) is included in Fig. 2. Note the varying intensities of direct emission of light in the combustion products. It is important to emphasize that in the current results, direct observation does not allow to visualize the presence of hydroxyl radical $\mathrm{OH}^{*}$ in the reaction zone of the combustion front because neither the polycarbonate nor the glass windows are transparent at the wavelength at which spontaneous emission occurs (i.e. $308 \mathrm{~nm}$ ). Available data on spectrometry suggests that the emission bands present in our results may come from the vibration-rotational spectrum of water, which lies in the visible range [10], [11].

Liveing and Dewar [12] showed that radiation from $\mathrm{H}_{2}$ flames increases with pressure, hence the re-illumination oscillations observed behind the front could be caused by a pressure increase during flame acceleration. Additionally, Schefer et al. [13] demonstrated that an increase in temperature only leads to an emission intensity increase without influencing the actual spectrum of the gas. Consequently, the spontaneous emission captured by the camera gives a slightly lower estimate of the true reactive front position. That said, from here on front position corresponds to the visible emission captured by the camera. 


\subsection{Parameters studied}

In this section, we describe each of the design parameters investigated. Namely, ignition energy, settling time between filling and ignition, material utilized for optical access, and ignition offset. For most experimental conditions, at least two tests were conducted to assess repeatability. A systematic comparison of all possible combinations among parameters would imply conducting around a hundred tests. Fractional factorial experimental design [14] was used to reduce the number of tests needed without compromising the validity of our conclusions to a resulting sample size of 41 (this includes the fact that some conditions were repeated up to four times). While we recognize that our sample size may not be large enough to draw meaningful conclusions on flame position variations induced by a specific parameter that lie below the standard deviation of the sample, our purpose here is to identify those with an appreciable effect on FA with the minimum number of tests possible. Additional details on this experimental matrix reduction methodology as well as its application to our study can be found in [14] and Appendix 1, respectively.

Ignition energy (igniter type and number of discharges): two different systems were used to ignite the mixture: (i) a commercial electric lighter (Pearl NX9440-944) connected to two electrodes, referred to as Igniter 1, and (ii) a discharge generator (Information Unlimited Ignitor10 HV Pulse Trigger) connected to the same electrodes, referred to as Igniter 2. Table 1 shows their technical specifications. Note that each igniter has a different frequency of operation (which determines the number of discharges produced when active) and energy per discharge, measured using a current (Lecroy CP031), and a voltage (B\&K Precision PR55) probes, wrapped around the wire feeding the electrodes. The measurement uncertainty reported is that obtained from the test-to-test variations while characterizing each igniter.

Table 1 Technical specification of igniters used.

\begin{tabular}{lll}
\hline & Igniter 1 & Igniter 2 \\
\hline Frequency $(\mathrm{kHz})$ & 16 & 0.5 \\
$E_{\min }$ & 44 discharges $\approx 0.28 \pm 0.02 \mathrm{~mJ}$ & 1 discharge $\approx 0.12 \pm 0.01 \mathrm{~mJ}$ \\
$E_{\max }$ & 287 discharges $\approx 1.45 \pm 0.08 \mathrm{~mJ}$ & 5 discharges $\approx 0.15 \pm 0.01 \mathrm{~mJ}$ \\
\hline
\end{tabular}

Figure 3 shows the time evolution of the ignition energy, $E$, for both igniters in stochiometric $\mathrm{H}_{2}$-air. Solid and dashed lines with symbols indicate their operation at the maximum, $E_{\max }$, and minimum, $E_{\min }$, ignition energy allowed by the igniters, respectively; the vertical dotted lines are visual aids showing the minimum/maximum flame arrival times to the open end of the channel over the entire dataset. It is worth noting that measurements were also performed in pure air and stoichiometric $\mathrm{H}_{2}-\mathrm{O}_{2}$ (not shown); these were found to behave similarly but their magnitudes differed by $\sim 15 \%$ (higher) and $\sim 45 \%$ (lower) 
for the former and latter mixtures considered. In line with the frequencies and $E_{\max }$ at which each igniter operates the number of discharges is visible for Igniter 2 but not for Igniter 1. The first discharge is the most energetic for both igniters, and exceeds the minimum ignition energy reported for stoichiometric $\mathrm{H}_{2}$-air mixtures with the same electrode spacing used in this study (i.e. $0.03 \mathrm{~mJ}$ at $1 \mathrm{~mm}$ spacing [15]).

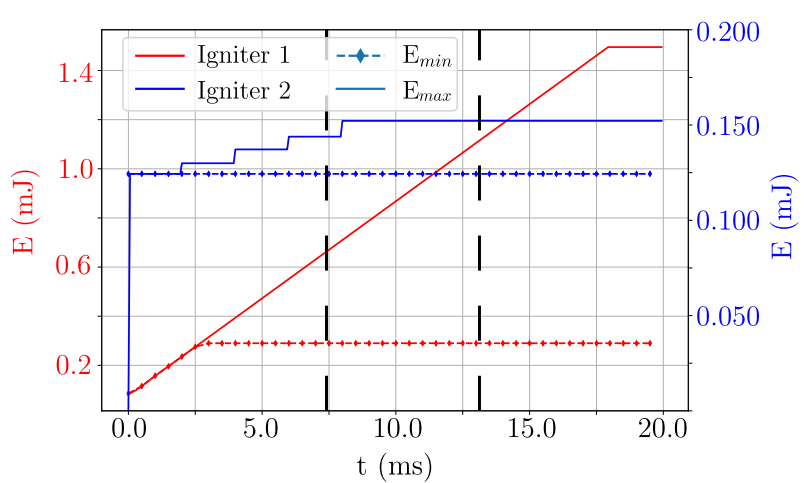

Fig. 3 Temporal evolution of ignition energy, $E$, for both igniters measured in stochiometric $\mathrm{H}_{2}$-air. Solid lines: operation at maximum ignition energy, $E_{\max }$; dashed lines with symbols: operation at minimum ignition energy, $E_{\min }$; vertical dashed lines: minimum/maximum flame arrival times to the open end of the channel over the entire dataset.

Settling time between end of filling and ignition: the cap closing the open-end of the channel was kept closed during $15 \mathrm{~s}$ or $60 \mathrm{~s}$ after the filling process to assess the effect of flow unsteadiness prior to ignition on FA. At the preset time the cap was removed from the open-end and one second later the mixture was ignited.

Material utilized for optical access: two different materials were tested, polycarbonate (plastic) and glass, both $8-\mathrm{mm}$ thick. The latter was held by an aluminum frame flushed on a flat seal ensuring air tightness and preventing direct contact between the glass and the aluminum frame (see Fig.4); the former was cut to conform to the dimensions of the aluminum block described in section 2.1. Although the optical properties and roughness characteristics of both materials are similar (i.e. arithmetical mean deviation of $\sim 0.03 \mu \mathrm{m}$; see [16] for details), plastic and glass do not share the same chemical properties. Plastic is a hydrocarbon that can combust and it is uncertain whether this can have an impact on the acquired chemiluminescence signal due to potential pyrolysis within the channel.

Ignition offset: due to manufacturing constraints an offset is present between the closed-end of the channel and the electrodes (see Fig.5). To assess its influence on FA, a removable plastic insert of $11 \mathrm{~mm} \times 10 \mathrm{~mm} \times 10 \mathrm{~mm}$ was 


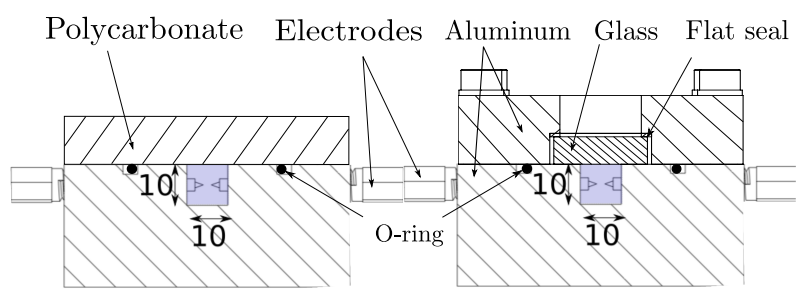

Fig. 4 Cross sectional view of channel showing polycarbonate (left) and glass (right) configurations.

placed behind the electrodes. In this manuscript, large ignition offset refers to cases without the plastic insert $\left(\sim 1.6 \mathrm{~cm}^{3}\right)$; whereas, a small ignition offset refers to cases in which the plastic insert is used $\left(\sim 0.4 \mathrm{~cm}^{3}\right)$.

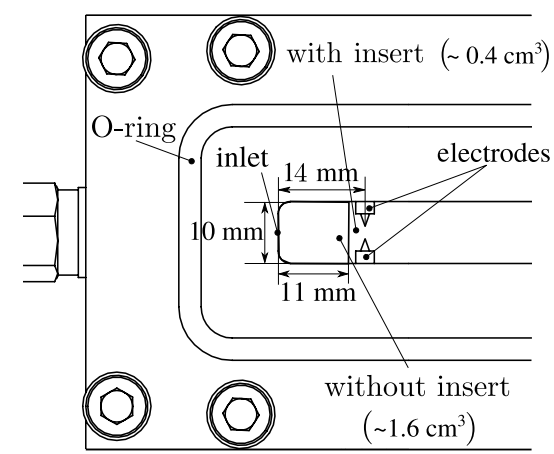

Fig. 5 Channel closed end showing detail to electrodes and ignition offset.

\section{Results}

Figures 6 and 7 are a compilation of luminous front positions data, i.e. $x-t$ diagrams. Colored bands represent the scatter obtained due to test-to-test variations, colored dashed lines show the mean position, $\bar{x}$, for the given sample, and black dashed lines show the mean position for the entire data set (computed using the means for each sample). The use of typical statistical metrics like variance and standard deviation would not be adequate to have a meaningful comparison among the parameters tested in the current context, instead, we define the absolute maximum deviation from the mean value, $d=|x-\bar{x}|_{\max }$, as follows: for each data point in a set, we compute the difference between the upper/lower bounds from the mean position and take the maximum. These values are reported in the plot next to the $x-t$ diagrams. Colored lines are intrinsic to a parameter, i.e., $d_{\text {par }_{1}}=\left|x_{\mathrm{par}_{1}}-\bar{x}_{\mathrm{par}_{1}}\right|_{\text {max }}$ or $d_{\mathrm{par}_{2}}=\left|x_{\mathrm{par}_{2}}-\bar{x}_{\mathrm{par}_{2}}\right|_{\max }$, whereas black lines are global, computed with the combined dataset presented 
on the left of the figure, i.e., $d_{\mathrm{g}}=\left|x_{\mathrm{par}_{(1+2)}}-\bar{x}_{\mathrm{par}_{(1+2)}}\right|_{\max }$. Having an intrinsic deviation of the same order or greater than that of the combined data set, i.e., $d_{\text {par }_{1}} \geq d_{\mathrm{g}}$ or $d_{\text {par }_{2}} \geq d_{\mathrm{g}}$, shows that the specific parameter considered does not influence flame propagation significantly. On the other hand, if the opposite holds, comparing the magnitude of the quantities $\left|d_{\mathrm{g}}-d_{\mathrm{par}_{1}}\right|_{\max }$ and $\left|d_{\mathrm{g}}-d_{\text {par }_{2}}\right|_{\text {max }}$ provides an estimate of the relative importance of the parameter investigated. The latter metric quantifies the dispersion of each dataset, and allows to identify potential inter-parameter interactions should the effect of a parameter be present in one configuration but not on the other. Response Surface Methods applied to the mean position of each data set, completes the analysis and allows to draw relevant conclusions on the inter-parameter interactions.

For clarity in the presentation and to illustrate the methodology, we only show results for two parameters in this section, the ignition energy and the ignition offset which were found to have a weak and strong influence on FA, respectively. The data for the remaining parameters tested (i.e. the settling time and the material utilized for optical access) are included in Appendix 2.

\subsection{Ignition energy (igniter type and number of discharges)}

While investigations on successive electric discharges have been conducted in channels to promote DDT via resonant shock wave amplification [17]-[19], our goal is to avoid igniter effects on flame acceleration.

To determine whether the successive electric discharges present in our setup are strong enough to perturb the unburned gas flow after ignition, we varied the number of discharges. Figure 6 -top shows the results using Igniter 1, a small ignition offset and plastic as the material for optical access, even though the flame reaches the end of the channel before the total energy deposition is completed (see Fig. 3), $\Delta E=E_{\max }-E_{\min }$ is larger with Igniter 1, which makes it more relevant to compare ignition energy levels. Overall, the front position evolution is similar for $t \leq 7.5 \mathrm{~ms}$ (for both datasets), and their scatter increases thereafter. Similarly, the respective front positions bifurcate after this time. Quantitatively, in terms of intrinsic deviations, $d_{E_{\min }}$ and $d_{E_{\max }}$, these are both less than $10 \mathrm{~mm}$ for $t \leq 7 \mathrm{~ms}$. For $7.5 \mathrm{~ms} \leq t \leq 10 \mathrm{~ms}, d_{E_{\min }}$ increases to $15 \mathrm{~mm}$ to finally reach $30 \mathrm{~mm}$ near the channel's open-end. The value of $d_{\mathrm{g}}$ is greater than $d_{E_{\min }}$ and $d_{E_{\max }}$ for most of this interval, indicating a potential influence of the ignition energy level on FA. The difference between the global deviation and the intrinsic deviations for maximum/minimum ignition energy are $\left|d_{\mathrm{g}}-d_{E_{\max }}\right|_{\max } \simeq 15 \mathrm{~mm}$ and $\left|d_{\mathrm{g}}-d_{E_{\min }}\right|_{\max } \simeq 10 \mathrm{~mm}$, respectively. The same tests performed with glass (not shown) do not show this bifurcation, as $d_{\mathrm{g}}$ always remained close to the intrinsic deviations, suggesting a potential interaction between parameters (i.e. ignition energy and material) or, alternatively, a negligible influence of the energy deposit.

Igniters 1 and 2 were tested using both energy levels to determine whether they perturb the flame propagation; the comparison is shown in Fig. 6-bottom. 


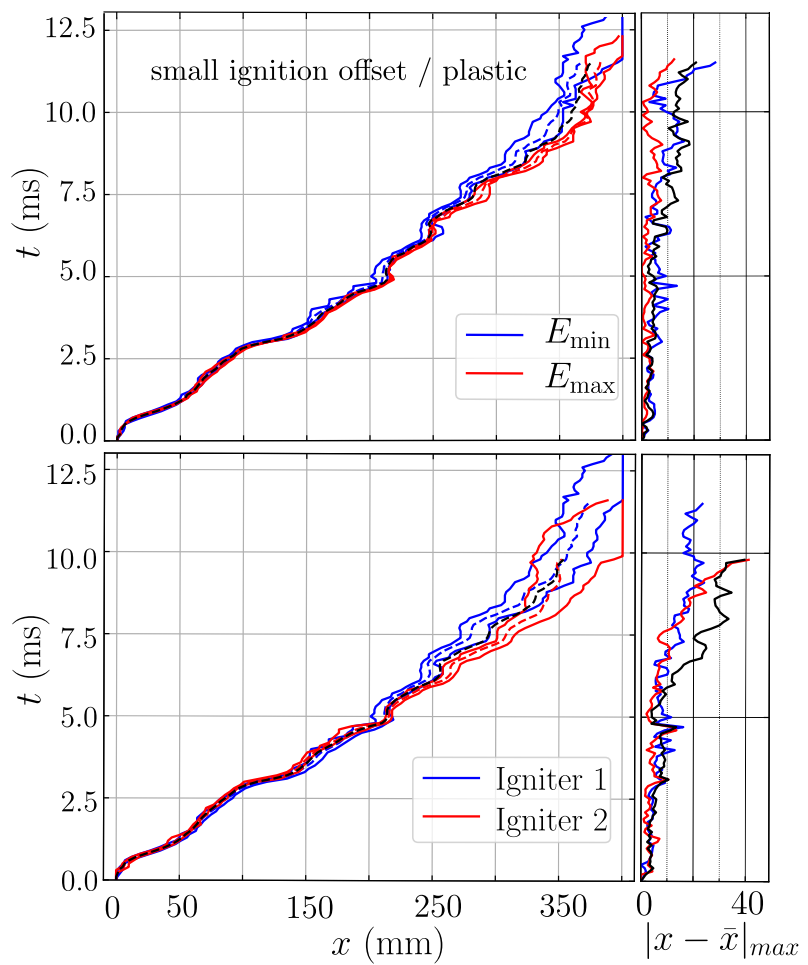

Fig. 6 Influence of total electric energy deposit (top), and igniter (bottom) on mean flame position, $\bar{x}$ (dashed line). Absolute maximum deviations from mean values, $|x-\bar{x}|_{\max }$. Top: Igniter 1 (sample size $=5$ tests), Bottom: both igniters, both energy levels (sample size $=$ 15 tests). Configuration: small ignition offset and plastic; conditions: stoichiometric $\mathrm{H}_{2}$-air at $p_{0}=100 \mathrm{kPa}$, and $T_{0}=290 \mathrm{~K}$

For $t \leq 5 \mathrm{~ms}$, both $d_{\mathrm{Ign}_{1}}$ and $d_{\mathrm{Ign}_{2}}$ are less than $15 \mathrm{~mm}$. For $5 \mathrm{~ms}<t<9 \mathrm{~ms}$, $d_{\mathrm{Ign}_{1}}$ and $d_{\mathrm{Ign}_{2}}$ both grow at a the same rate. At $t>9 \mathrm{~ms}, d_{\mathrm{Ign}_{2}}$ increases to $40 \mathrm{~mm}$, showing a larger deviation than Igniter 1 . The intrinsic discrepancies are stronger near the open end but are of the same order as $d_{\mathrm{g}}$. A potential effect of igniter type on FA can be seen for $6 \mathrm{~ms}<t<9 \mathrm{~ms}$, when $d_{\mathrm{g}}$ is approximately twice the value of $d_{\mathrm{Ign}_{1}}$ and $d_{\mathrm{Ign}_{2}}$. The difference between the global deviation and the intrinsic deviations for igniter 1 /igniter 2 are $\mid d_{\mathrm{g}}-$ $\left.d_{\mathrm{Ign}_{1}}\right|_{\max } \simeq 20 \mathrm{~mm}$ and $\left|d_{\mathrm{g}}-d_{\mathrm{Ign}_{2}}\right|_{\max } \simeq 18 \mathrm{~mm}$, respectively. While in both cases $d_{\mathrm{g}}$ is greater than their corresponding intrinsic deviations, the difference is larger and occurs over a longer period when comparing igniters than total energy deposit (see Fig. 6) which conclusively shows that the igniter type (i.e. discharge frequency) has a stronger influence on FA. In summary, the energy deposit and discharge power used do not perturb significantly the flame propagation, but the discharge frequency of the different igniters seem to have a moderate impact. 
3.2 Ignition offset

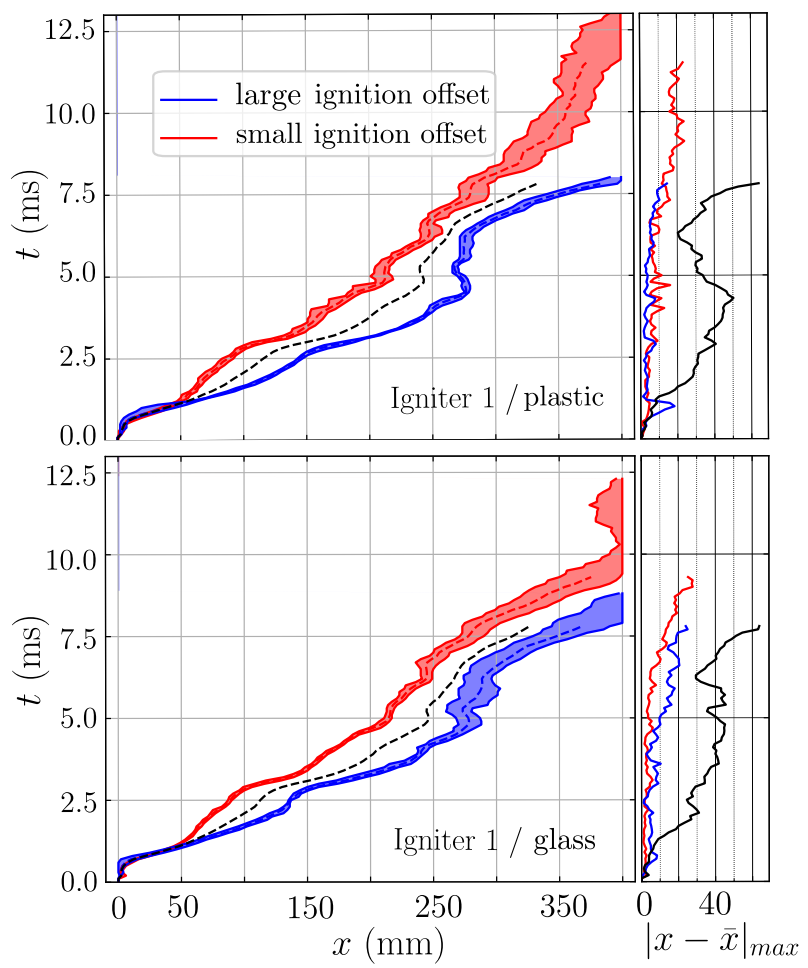

Fig. 7 Influence of ignition offset on mean flame propagation $\bar{x}$ (dashed line). Absolute maximum deviation from mean value $|x-\bar{x}|_{\max }$. Top: plastic (sample size $=10$ tests), Bottom: glass (sample size $=12$ tests). Configuration: Igniter 1 for both figures; conditions: stoichiometric $\mathrm{H}_{2}$-air at $p_{0}=100 \mathrm{kPa}$, and $T_{0}=290 \mathrm{~K}$.

The front position data comparing the two different ignition offsets described in section 2.4 is presented in Fig. 7. Results were collected with plastic and glass as the material for optical access and are presented as the top and bottom plots, respectively. There is a distinct bifurcation in the data occurring at $(t, x)=(1 \mathrm{~ms}, 50 \mathrm{~mm})$. The intrinsic deviations for large ignition offset and small ignition offset, $d_{\mathrm{LIO}}$ and $d_{\mathrm{SIO}}$, do not surpass $30 \mathrm{~mm}$ throughout for the tests performed with glass, and exhibit a higher deviation than plastic with a small ignition offset. Finally, note that $d_{\mathrm{SIO}}$ and $d_{\text {LIO }}$ for $t>1 \mathrm{~ms}$ always lie below the global deviation, $d_{\mathrm{g}}$, suggesting a strong dependence of flame propagation and subsequent acceleration on this parameter. The difference between the global deviation and the instrinsic deviations for small/large ignition offsets are $\left|d_{\mathrm{g}}-d_{\mathrm{SIO}}\right|_{\max } \sim 55 \mathrm{~mm}$ and $\left|d_{\mathrm{g}}-d_{\mathrm{LIO}}\right|_{\max } \sim 50 \mathrm{~mm}$, significantly larger than those obtained for ignition energy. 


\subsection{Controlling interactions}

Based on the results above, as well as those shown in Appendix 2 (sections 2.1 and 2.2), there seems to be some inter-parameter interactions. Namely, ignition energy and material, as well as the ignition offset and material. A more indepth analysis is thus needed to confirm these observations. Response Surface Methods for design of experiments [14], [20] can be applied to build a response model to highlight the effect of each parameter, and their interactions. From this model a vector of coefficients, $\boldsymbol{a}$, is obtained whose elements allow to evaluate the influence of a particular parameter, $a_{1}$ for parameter 1 , or the interactions between different parameters, $a_{12}$ for the interaction of parameter 1 with parameter 2 , and so on, on the response (i.e. experimental output). Instead of directly computing these coefficients to construct a response surface (which can result in a large number of tests), the model can be simplified by neglecting high order interactions, among three or more parameters for example (which are less likely), and by using aliases, $\boldsymbol{l}$, in which the different experimental factors are confounded based on the experimental matrix reduction methodology described in [14]. Finally, using the mean front position, $\bar{x}$, as our response we can solve for vector $\boldsymbol{l}$ and plot the temporal evolution of its elements as shown in Fig. 8. Subscripts 1,2, 3 and 4 correspond to the settling time, ignition offset, material and ignition energy, respectively; the remaining subscripts are interactions among parameters. Additional details on the mathematical framework used are included in Appendix 1.

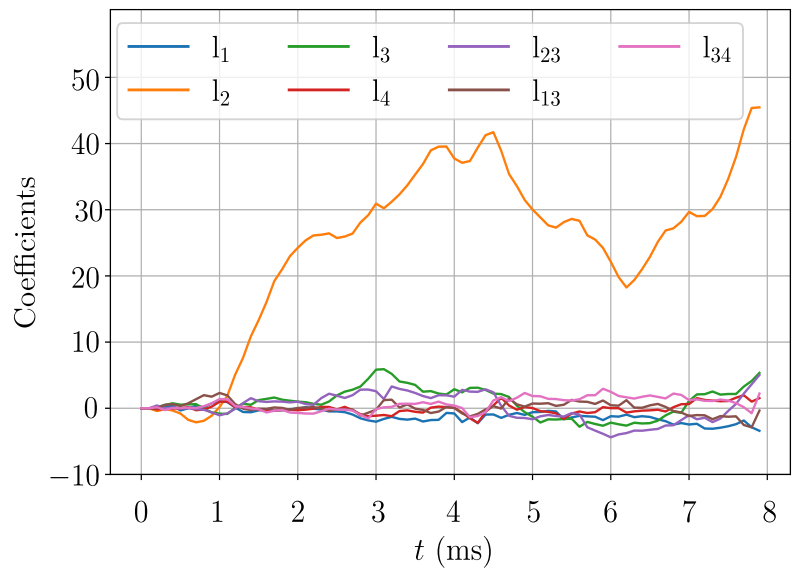

Fig. 8 Temporal evolution of alias coefficients, $l_{i}$, for a $2^{(n-1)}$ fractional design. Subscripts 1: settling time, 2: ignition offset, 3: material, 4: energy; 23, 13, 34: refer to inter-parameter interactions. Configuration: Igniter 1 ; conditions: stoichiometric $\mathrm{H}_{2}$-air at $p_{0}=100 \mathrm{kPa}$, and $T_{0}=290 \mathrm{~K}$.

The results for $l_{2}$ show the influence of ignition offset and combined interaction between the settling time, and ignition energy, $E$, on the recorded flame position. Its value lies significantly above the rest of the aliases plotted. $l_{1}$ 
shows the influence of settling time together with the combined interaction of ignition offset and igntion energy whereas $l_{4}$ shows that of ignition energy and the combined interaction of settling time and ignition offset. Both coefficients attain values of less than 3 suggesting that settling time and ignition energy can be neglected, and that only the ignition offset has a significant influence on the front position. The window material ranks second (i.e. $l_{3, \max } \approx 5.8$ ). The interaction of window material and ignition offset ranks third attaining a value of $l_{23, \min } \approx-5.2$. Finally, the interaction of settling time and window material, and window material and $E$ are not significant with $l_{13} \approx 3$ and $l_{34} \approx 3.2$, respectively.

\section{Discussion}

The analyses that follow focus on the ignition offset, as it was the only parameter to show a significant influence on flame propagation, and aim to provide explanations to the discrepancies observed between small and large ignition offsets.

\subsection{Early stages of flame propagation}

The flame front position data was found to be very similar between small and large ignition offset for the first $50 \mathrm{~mm}$ of propagation (see Fig. 7); thereafter there was a clear bifurcation with the large ignition offset $\mathrm{H}_{2}$-air data showing increased acceleration rates. It is thus necessary to analyze the early stages of flame propagation to explain the source of the discrepancy. We will use the theory developed in [21] for this purpose. Figure 9 shows a comparison of the flame topologies obtained with small and large ignition offsets using contours of luminous emission.

Five distinct stages can be identified: (i) a hemispherical flame kernel that transits to a finger flame $(t=0.4 \mathrm{~ms})$; (ii) interaction of the flame skirt with the walls resulting in front deceleration $(t=0.8 \mathrm{~ms}$ ); (iii) flattening of the flame surface $(t=1 \mathrm{~ms})$; (iv) inversion $(t=1.1 \mathrm{~ms})$, and $(\mathrm{v})$ formation of a tulip flame. Such observations have been previously reported [9], [21]-[24], and theoretical models capable of accurately describing the experimentally observed early stages of flame propagation have been developed [21], [24].

The normalized flame front position evolution for a planar geometry is given by [21]:

$$
\frac{x_{\mathrm{tip}}}{L}=\frac{2 \Theta_{1}\left[\exp \left(\sigma_{2} \tau\right)-1\right]}{\left(\sigma_{2}-\sigma_{1}\right) \exp \left(\sigma_{2} \tau\right)+\left(\sigma_{2}+\sigma_{1}\right)}
$$

where,

$$
\Theta_{1}=\Theta-\operatorname{Ma}(\gamma-1)(\Theta-1)^{2} ; \quad \tau=\frac{t S_{L}}{L}
$$




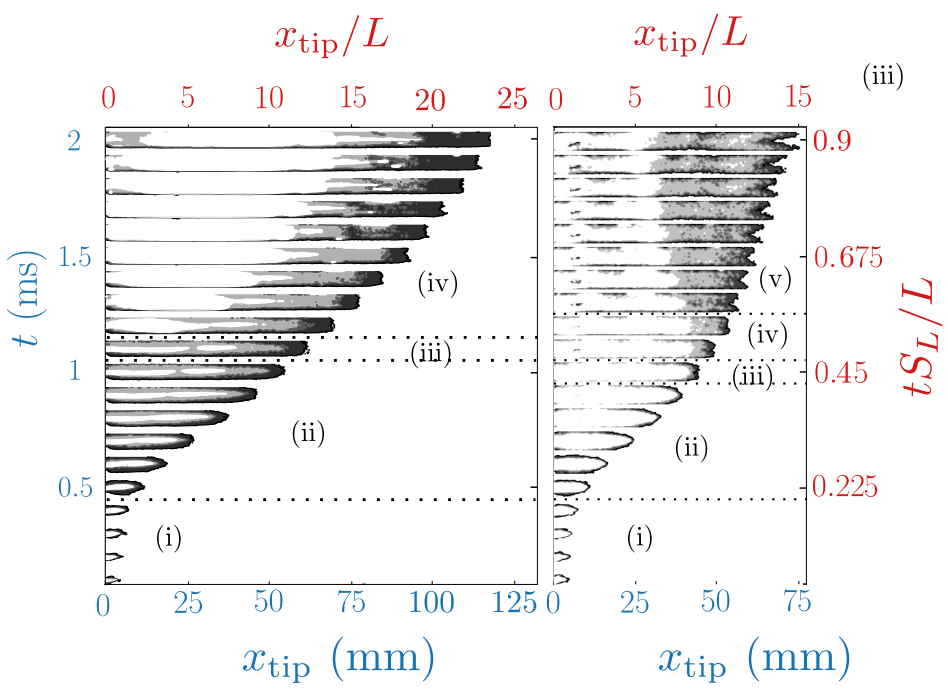

Fig. $9 x-t$ diagrams for early stages of flame propagation. Inverted color experimental images showing flame topology stages. Left: large ignition offset, Right: small ignition offset. Configuration: plastic and igniter 2; conditions: stoichiometric $\mathrm{H}_{2}$-air at $p_{0}=100 \mathrm{kPa}$, and $T_{0}=290 \mathrm{~K}$. Electrodes at $x=0, \Delta \mathrm{t}: 0.1 \mathrm{~ms}$.

$$
\begin{gathered}
\sigma_{1}=(\Theta-1)[1-\operatorname{Ma}(\Theta+2(\gamma-1)(\Theta-1))] \\
\sigma_{2} \equiv \sqrt{\sigma_{1}^{2}+4 \operatorname{Ma\gamma } \Theta_{1}(\Theta-1)^{2}}
\end{gathered}
$$

In Eqns. 2 to $4, \mathrm{Ma}=S_{L} / c_{o}$ is the flame Mach number with $c_{o}$ being the speed of sound in fresh reactants, $\Theta$ is the expansion ratio, $\gamma$ is the ratio of specific heats, and $L$ is a length scale taken to be the half-height of the channel in our case.

Two non-dimensional characteristic times are defined [21]:

$$
\tau_{\text {sph }}=\frac{1}{\Theta-1} ; \quad \tau_{\text {wall }}=\frac{\ln \Theta}{\Theta-1}\left[1+\operatorname{Ma}(\gamma-1)\left(\Theta^{2}-1\right)\left(1-\frac{\Theta-1}{\Theta \ln \Theta}\right)\right]
$$

$\tau_{\text {sph }}$ is the elapsed time from ignition in which the flame expands as a spherical kernel; and $\tau_{\text {wall }}$ is the time at which the flame first interacts with the channel's walls. For $\mathrm{Ma}=0.0055, S_{L}=2.25 \mathrm{~m} / \mathrm{s}, \Theta=6.853, \gamma=1.4$, $c_{o}=409 \mathrm{~m} / \mathrm{s}$ and $L=0.005 \mathrm{~m}$, the values corresponding to our configuration, we obtained the results summarized in Table 2 . These values are compared with those experimentally measured, $t_{\mathrm{sph}}$ and $t_{\text {wall }}$, which are determined by pin-pointing the first sign of deformation in the flame, and by taking the maximum velocity recorded during early stages of flame propagation; better temporal resolution and improved visualization techniques would give more 
Table 2 Comparison of experimental and theoretical [21] characteristic times for early stages of flame propagation.

\begin{tabular}{llll}
\hline Characteristic times & Theory & small ignition offset & Experimental \\
& & & large ignition offset \\
\hline$\tau_{\mathrm{sph}}\left(t_{\mathrm{sph}}\right)$ & $0.17(0.38 \mathrm{~ms})$ & $0.18(0.4 \mathrm{~ms})$ & $0.18(0.4 \mathrm{~ms})$ \\
$\tau_{\text {wall }}\left(t_{\mathrm{wall}}\right)$ & $0.35(0.77 \mathrm{~ms})$ & $0.32(0.7 \mathrm{~ms})$ & $0.35(0.78 \mathrm{~ms})$ \\
$u_{f, \max }$ & $80 \mathrm{~m} / \mathrm{s}$ & $75 \mathrm{~m} / \mathrm{s}$ & $90 \mathrm{~m} / \mathrm{s}$ \\
\hline
\end{tabular}

accurate measurements of the experimental times. The values reported are mean quantities from the available data set.

Figure 10 shows the experimental flame front position compared with the prediction of Eq. (1). The bands show upper/lower bounds of the experimental data. A few observations include: (i) theory and experiment are in good agreement for $t<1 \mathrm{~ms}$; (ii) deceleration of the front occurs earlier for small ignition offset than large ignition offset; (iii) for $t>1 \mathrm{~ms}$, three-dimensional effects (i.e. corners not accounted for in a planar description of the flow) may start playing a role in the flame dynamics; (iv) at $t \sim 3 \mathrm{~ms}$ the flame is observed to re-accelerate in both cases.

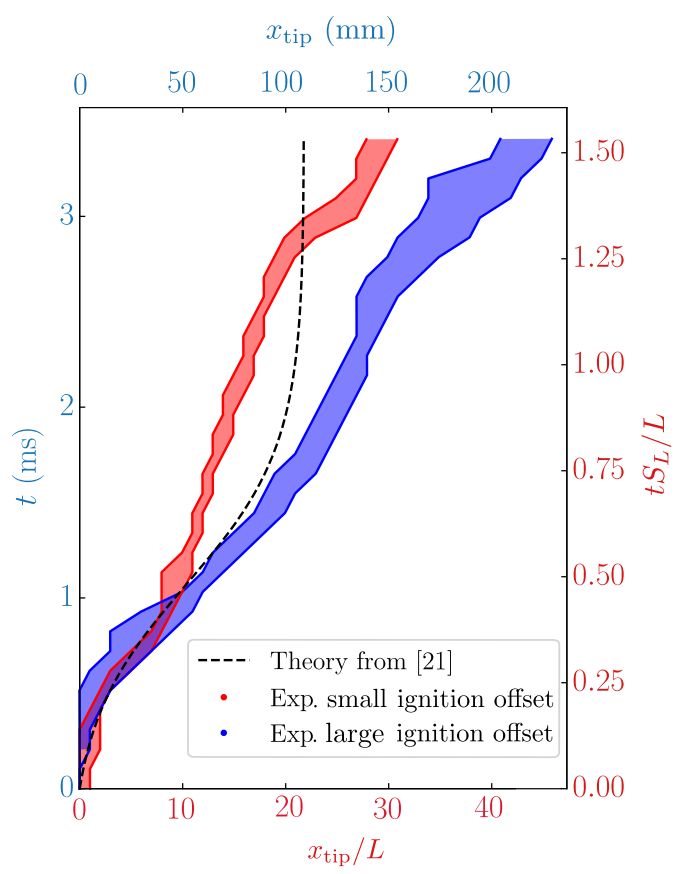

Fig. $10 x-t$ diagram for early stages of flame propagation. Theoretical predictions and comparison with experimental data. Scaled flame tip $\left(x_{\text {tip }} / L\right)$ position versus non-dimensional time $\left(t S_{L} / L\right)$. Conditions: stoichiometric $\mathrm{H}_{2}$-air at $p_{0}=100 \mathrm{kPa}$, and $T_{0}=290 \mathrm{~K}$. Electrodes at $x=0$; temporal resolution between frames is $\Delta t=0.1 \mathrm{~ms}$ 
A simple acoustic model allows to rationalize the aforementioned observations. For large ignition offset, the distance from the electrodes to the closed end of the channel is $14 \mathrm{~mm}$ (see Fig. 5). Using $u_{f}=\Theta S_{L}=15.42 \mathrm{~m} / \mathrm{s}$ as a first order estimate for the flame velocity in the laboratory frame of reference, it takes $0.9 \mathrm{~ms}$ for the flame to reach the closed end. The interaction with the wall will result in a perturbation propagating at the local speed of sound in burnt products, $c_{b}=1010 \mathrm{~m} / \mathrm{s}$. From Fig. 10 the bifurcation in the data occurs at $x=50 \mathrm{~mm}$. Hence the time for a perturbation to travel from the closed end to the front position $(\Delta x=64 \mathrm{~mm})$ is $0.064 \mathrm{~ms}$; the expected interaction with the flame front should then occur at $t \sim 0.964 \mathrm{~ms}(\tau=0.45)$ which is in agreement with the observed increase in flame speed for the large ignition offset case. Flame inversion is thus delayed.

The re-acceleration at $t \sim 3 \mathrm{~ms}(\tau=1.35)$ can be explained using the same rationale. The acoustic perturbations that originate from the finger flame acceleration at $t_{\text {wall }}$ travel back from the open end and reflect from the closed end to give an additional "boost" to the flame. The one-dimensional analysis of Kurylo [25] takes this acoustic feedback as one of the FA mechanisms at early stages. Previous experimental [26] and numerical [27] results showed that flame inversion is a hydrodynamic process which is influenced by both boundary conditions and acoustics (even if the latter is not a necessary condition for flame inversion according to [28]). This hydrodynamic process is likely perturbed by acoustic waves that travel from the closed end which may explain the observed discrepancy with the theory. In spite of the overall agreement observed between our simple acoustic model and our experimental results, we note that the expression used above for $u_{f}$ overestimates the flame propagation velocity towards the close end and, hence, the time at which the perturbation is produced. In fact, the flame front-induced flow quickly stagnates, due to the presence of the end wall, resulting in a continuously decreasing fuel consumption rate that would eventually equal its burning velocity (i.e. $u_{f} \sim S_{L}$ ). Most of the products expansion occurs towards the open-end thereby accelerating the front in that direction. The role of acoustic perturbations in the postulated delay of flame inversion for large ignition offsets at early stages of FA will be investigated in more detail via a thorough analysis of schlieren images and numerical simulations in future. The additional oscillations observed during the later stages of the FA process, and the increased scatter at the end of the channel is discussed in subsection 4.2 .

\subsection{Front velocity and acceleration}

In this section we compute flame velocity and acceleration from the flame position experimental data. A Savistzky-Golay filter is applied to smooth the data, and numerical differentiation is used to obtain corresponding instantaneous velocities; the resulting data is once again filtered and differentiated to compute accelerations. Figure 11 shows velocity (top) and acceleration (bottom) evolution comparing igniters 1 and 2 for small ignition offset and using 
plastic as the material for optical access. Similar to the front position data, results are repeatable during the first few milliseconds $(t<8 \mathrm{~ms})$, and the scatter increases thereafter. The oscillations period of the flame velocity and acceleration match regardless of igniter type and number of discharges. Figure 12 shows the same data comparing small and large ignition offsets for Igniter 2 and plastic as the material utilized for optical access. During the first $4 \mathrm{~ms}$ oscillation periods seem to be in-phase where only amplitudes are affected; for $t>4 \mathrm{~ms}$ large ignition offset exhibits earlier oscillatory behavior.
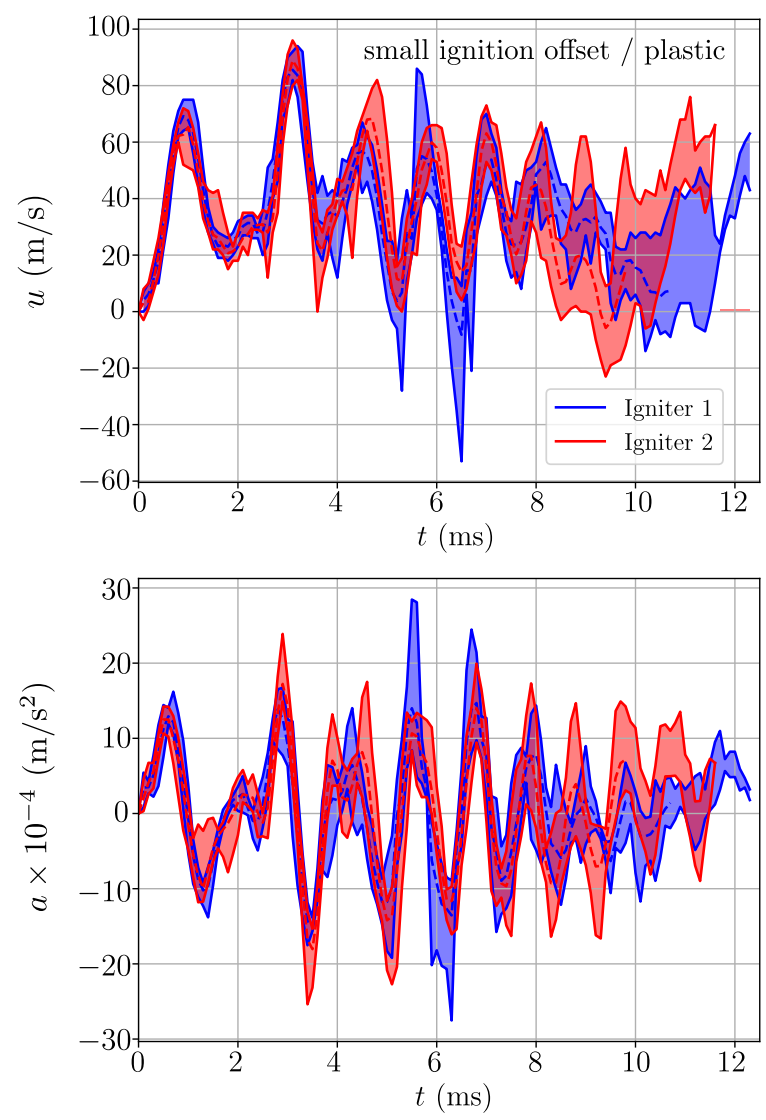

Fig. 11 Front velocity and acceleration. Configuration: Small ignition offset and plastic; conditions: stoichiometric $\mathrm{H}_{2}$-air at $p_{0}=100 \mathrm{kPa}$, and $T_{0}=290 \mathrm{~K}$.

For all small ignition offset cases shown, front velocities stay below $100 \mathrm{~m} / \mathrm{s}$, in contrast, the large ignition offset cases attain larger peaks (up to $\sim 150 \mathrm{~m} / \mathrm{s}$ as seen in Fig. 12) likely due to the acoustic "boost" discussed in section 4.1; similar velocity records were obtained in [9] using the same mixture but with a larger cross section $(82 \times 82 \mathrm{~mm})$. Note that the magnitude of all peaks is 

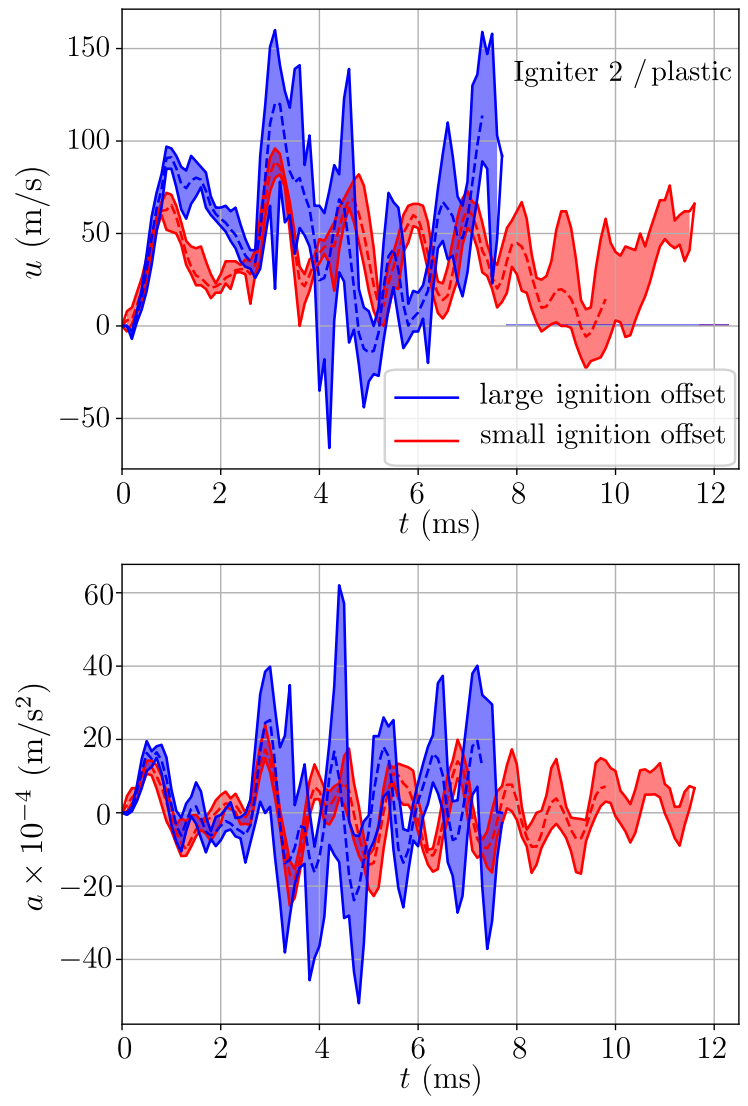

Fig. 12 Front velocity and acceleration. Configuration: Igniter 2 and plastic; conditions: stoichiometric $\mathrm{H}_{2}$-air at $p_{0}=100 \mathrm{kPa}$, and $T_{0}=290 \mathrm{~K}$.

larger for large ignition offset and exhibit no signs of decay throughout the tests, whereas for small ignition offset the amplitude of the oscillations decay progressively. The lowest amplitude recorded corresponds to the fourth peak $(60 \mathrm{~m} / \mathrm{s}$ at $t=5.5 \mathrm{~ms})$, and is directly associated with the "stagnation" observed in Fig 7 for large ignition offset. Subsequently, the front goes through a shorter deceleration and a longer acceleration at rates similar to those observed for small ignition offset, resulting in front velocities that are two times higher towards the end of the channel for large than small ignition offsets. The differences alluded to above for the upper/lower bounds of the velocities and accelerations recorded become less prominent once the mean values are compared (see dashed lines in Figs. 11 and 12). The velocity magnitudes recorded with small ignition offsets are of the same order as those obtained in [29], [30] using longer channels and different initial and boundary conditions (i.e. sub-atmospheric pressures, closed and partially open channels). Surprisingly, limited front position, velocity and acceleration data seems to be available 
for setups as that considered here. This data once again shows the strong influence of ignition offset in the flame propagation and subsequent acceleration. Comparable evolution has been described in previous work investigating the oscillatory behavior of flames propagating in circular tubes and square cross-section channels [31]-[36] with the same boundary conditions used here. Their results provide evidence that reflection of acoustic waves from the open end perturb the flow, and the flame, in turn, responds to these perturbations. While coupling between oscillations and pressure waves within channels has been previously shown, it remains unclear if acoustics alone is responsible for the observed oscillations or if they simply contribute to trigger intrinsic flame instabilities. In numerical simulations of flame propagation assuming non-reflecting boundary conditions [28], oscillations due to flame inversion were identified showing that there is an additional source of oscillations different from acoustic feedback in these type of configurations. Furthermore, in [35], the authors compared predicted modes of oscillations due to acoustics with experimental results and found discrepancies that were attributed to the assumed reflection/transmission coefficients. On the other hand, the work in [34], showed that the observed oscillations depend on tube length and sound speed, showing a clear influence of acoustics. The ignition offset changes the oscillation periods in a similar manner by increasing the effective length of the channel.

\subsection{Observed oscillations during FA}

To further understand our data and find the reason of the increased scattering as the open end is approached, we performed schlieren visualization at the channel exit. This allowed to highlight characteristic structures, similar to what was observed in [37]. Successive toroidal vortices (or puffs) of varying intensities carrying reactive mixture emanate from the open end prior to flame arrival. Figure 13 shows an example of the flow structures observed including shear layers and diffuse interfaces; the raw schlieren images were enhanced through background subtraction. From the videos obtained, fresh gas seems to be drawn inside the channel after each puff.

By placing a virtual probe at the outlet (red line in Fig. 13), we recorded the puff time history shown in Fig. 14; the arrows in the latter figure correspond to the puffs shown in Fig. 13.

Using a simple acoustic wave propagation model, in Fig. 15 we track the perturbations induced by the maximum velocity peaks observed in Fig. 11 assuming a constant sound speed in fresh mixture $\left(c_{o}=409 \mathrm{~m} / \mathrm{s}\right)$. Blue squares indicate the location of velocity peaks, blue dashed lines the theoretical acoustic perturbation paths, and green squares the puffs detection from Fig. 14. On the right of Fig. 15, the $x-t$ diagram obtained from schlieren visualization shows the path of puffs outside the channel traveling at $\sim 85 \mathrm{~m} / \mathrm{s}$ then decaying to $\sim 60 \mathrm{~m} / \mathrm{s}$; waves traveling at Ma $\sim 1$ were also observed and are indicated as black squares in the figure; finally, the red square indicates the arrival of the 


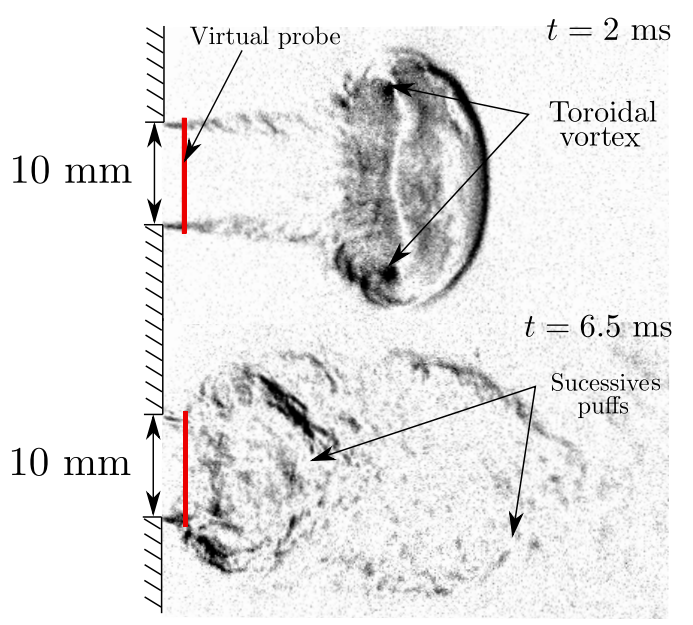

Fig. 13 Puffs observed at the end of the channel using schlieren visualization.

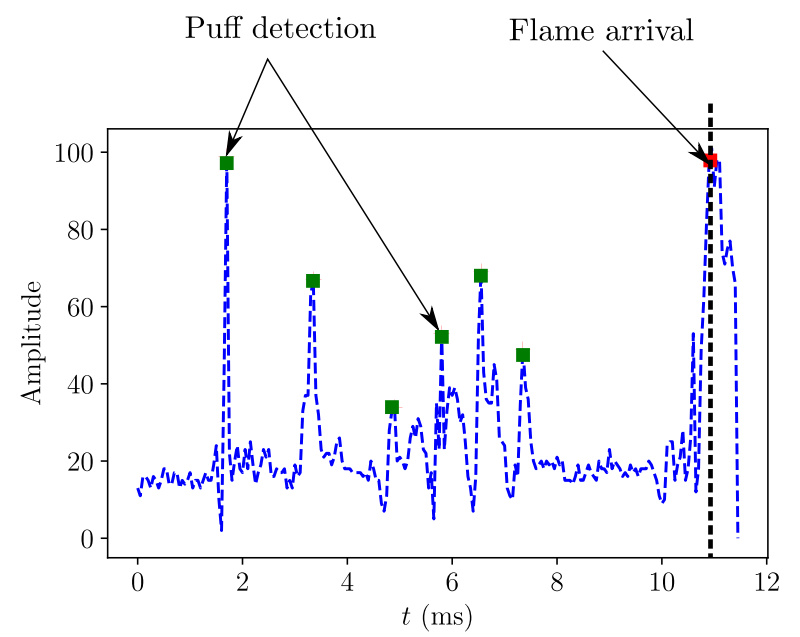

Fig. 14 Puffs time history for small ignition offset. Green squares indicate the pulses due to the puffs across the virtual probe; the red square and black dashed line indicate the flame arrival time.

flame. While the theoretical perturbation path matches the observation of the first and second puffs, subsequent puffs occur later, which implies this perturbation travels slower (at $\sim 200 \mathrm{~m} / \mathrm{s}$ ). The flow induced by the perturbations generate peaks of flow rate taking the form of puffs when reaching the open end. Upon reflection of the pressure perturbation, ambient mixture is drawn inside the channel, changing the composition of the unburned mixture close to the open end resulting in an in-flow. This may explain the delay observed upon subsequent puffs arrival times, and the scattering of front position near 


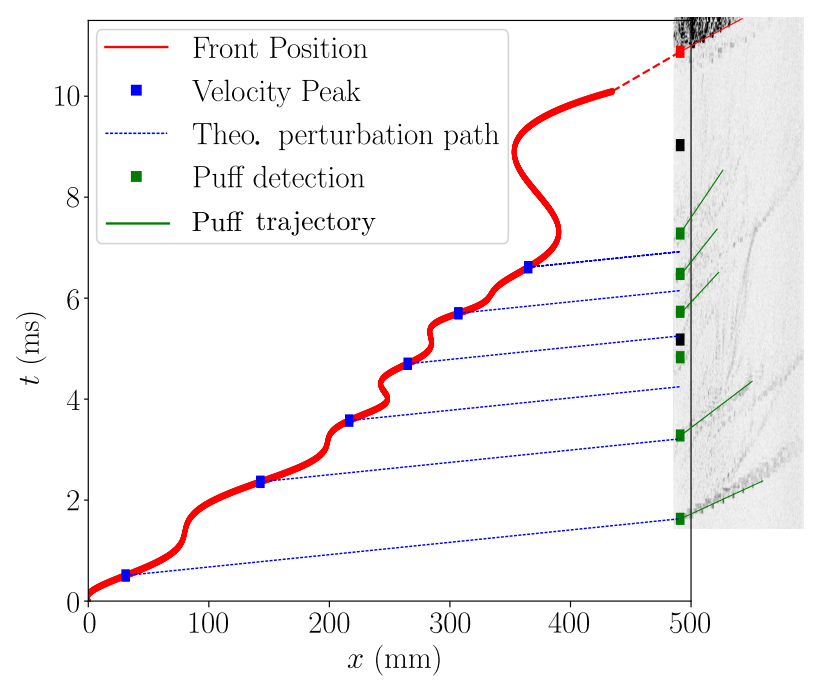

Fig. $15 x-t$ diagram for small ignition offset. Blue squares are the times at which velocity peaks are detected from the front velocity analysis in Fig. 12, blue dashed lines are the theoretical acoustic perturbation paths, green squares indicate the pulses due to the puffs detected across the virtual probe, and the red square shows the flame arrival time.

the end of the channel observed throughout our experimental campaign (See Figs. 6 to 18).

For completeness, the influence of the ignition offset for a mixture in which DDT is theoretically possible for the available channel length (i.e. $\mathrm{H}_{2}-\mathrm{O}_{2}$ ) is shown in Appendix 3.

\section{Conclusion and perspectives}

Stoichiometric $\mathrm{H}_{2}$-air flame propagation experiments were conducted using a 10 -mm $\times 10$-mm square cross-section channel of 482-mm in length. Direct observation was used to investigate the flame dynamics; the limitations of this visualization technique to study flame acceleration in $\mathrm{H}_{2}$ mixtures were highlighted. The effect of facility specific parameters (i.e. ignition energy, boundary conditions near the ignition end, material utilized for optical access and settling time between filling and ignition) on the recorded flame positions was assessed. Analysis of our results, based on factorial experimental design and response surface methods, conclusively showed that the boundary condition near the ignition end (small/large ignition offset relative to the electrodes position) was found to have the largest influence on flame propagation and acceleration with the remaining parameters playing a minor role. The ignition offset significantly influences the early stages of propagation (flame inversion) and subsequent flame dynamics (acoustics). The regions of our data where the largest scatter was observed (i.e. ignition- and open-end) were analyzed through the use of existing theories or simple acoustic models; the oscilla- 
tions recorded during propagation were linked to the flow rate dynamics at the channel exit. The increased test-to-test variability towards the end of the channel seems to be the result of cold inert gas drawn inside the channel after each puff and associated reduced mixture reactivity. Small ignition offsets are recommended to reduce the oscillations observed during flame acceleration.

Lastly, two important practical final remarks: (i) this study provides a detailed account of the design parameters to consider when designing a small scale experimental facility to study flame acceleration, and highlights the role played by the ignition offset on the flame dynamics. To the authors' knowledge, this has not been documented before as presented; (ii) the computed front velocities provide new data (as it seems to be rather scarce for the length scale, and stoichiometry considered) to assess flame acceleration hazards and/or inform design/manufacturing strategies in industries that store/handle hydrogen.

Acknowledgements The authors gratefully acknowledge l'École Doctorale SIMME, and the contribution of the technical personnel at l'Institut Pprime.

\section{References}

[1] R. Zipf Jr, V. Gamezo, M. Sapko, W. Marchewka, K. Mohamed, E. Oran, D. Kessler, E. Weiss, J. Addis, F. Karnack, et al., "Methane-air detonation experiments at niosh lake lynn laboratory," Journal of Loss Prevention in the Process Industries, vol. 26, no. 2, pp. 295-301, 2013. DOI: https://doi.org/10.1016/j.jlp.2011.05.003.

[2] G. Ciccarelli and S. Dorofeev, "Flame acceleration and transition to detonation in ducts," Progress in energy and combustion science, vol. 34, no. 4, pp. 499-550, 2008. DOI: https ://doi.org/10.1016/j.pecs. 2007.11 .002$.

[3] P. Wolański, "Detonative propulsion," Proceedings of the Combustion Institute, vol. 34, no. 1, pp. 125-158, 2013. DOI: https://doi.org/10 . 1016/j.proci.2012.10.005.

[4] S. B. Dorofeev, M. Kuznetsov, V. Alekseev, A. Efimenko, and W. Breitung, "Evaluation of limits for effective flame acceleration in hydrogen mixtures," Journal of loss prevention in the process industries, vol. 14, no. 6, pp. 583-589, 2001. DOI: https : //doi .org/10 .1016/S09504230 (01) 00050-X.

[5] M. Kuznetsov, I. Matsukov, V. Alekseev, W. Breitung, and S. Dorofeev, "Effect of boundary layer on flame acceleration and DDT," in 20th Int. Colloq. on the Dynamics of Explosions and Reactive Systems (ICDERS), McGill University, Montreal, Canada, vol. 31, 2005.

[6] M. Kuznetsov, M. Liberman, and I. Matsukov, "Experimental study of the preheat zone formation and deflagration to detonation transition," Combustion Science and Technology, vol. 182, no. 11-12, pp. 1628-1644, 2010. DOI: https://doi.org/10.1080/00102202.2010.497327. 
[7] V. Gamezo, A. Khokhlov, and E. Oran, "Effects of wakes on shockflame interactions and deflagration-to-detonation transition," Proceedings of the Combustion Institute, vol. 29, no. 2, pp. 2803-2808, 2002. DOI: https: //doi .org/10.1016/S1540-7489(02)80342-X.

[8] E. Dziemińska and A. K. Hayashi, "Auto-ignition and DDT driven by shock wave-boundary layer interaction in oxyhydrogen mixture," International Journal of Hydrogen Energy, vol. 38, no. 10, pp. 4185-4193, 2013. DOI: https://doi.org/10.1016/j.ijhydene.2013.01.111.

[9] H. Xiao, Q. Duan, L. Jiang, and J. Sun, "Effects of ignition location on premixed hydrogen/air flame propagation in a closed combustion tube," International Journal of Hydrogen Energy, vol. 39, no. 16, pp. 8557-8563, 2014. DOI: https://doi.org/10.1016/j.ijhydene.2014.03.164.

[10] T. Kitagawa, "Emission spectrum of the oxy-hydrogen flame and its reaction mechanism:(i) formation of the activated water molecule in higher vibrational states," The Review of Physical Chemistry of Japan, vol. 13f, no. 2, 1939.

[11] A. G. Gaydon, "Flame spectra in the photographic infra-red," Proceedings of the Royal Society of London. Series A. Mathematical and Physical Sciences, vol. 181, no. 985, pp. 197-209, 1942. DoI: https://doi.org/ 10.1098/rspa.1942.0071.

[12] G. Liveing and J. Dewar, "On the influence of pressure on the spectra of flames," Astronomy and Astro-Physics (formerly The Sidereal Messenger), vol. 11, pp. 215-223, 1892. DOI: https://doi.org/10.1098/ rspl.1890.0086.

[13] R. W. Schefer, W. D. Kulatilaka, B. D. Patterson, and T. B. Settersten, "Visible emission of hydrogen flames," Combustion and flame, vol. 156, no. 6, pp. 1234-1241, 2009. DOI: https : / / doi .org/10 . 1016/j . combustflame.2009.01.011.

[14] J. Goupy, Plans d'expériences. Ed. Techniques Ingénieur, 2006.

[15] R. Ono and T. Oda, "Spark ignition of hydrogen-air mixture," Journal of Physics: Conference Series, vol. 142, p. 012 003, Dec. 2008. DoI: https: //doi.org/10.1088/1742-6596/142/1/012003.

[16] G. Huber, S. N. Gorb, N. Hosoda, R. Spolenak, and E. Arzt, "Influence of surface roughness on gecko adhesion," Acta biomaterialia, vol. 3, no. 4, pp. 607-610, 2007. DOI: https://doi .org/10.1016/j .actbio. 2007 . 01.007.

[17] S. Frolov, V. Y. Basevich, V. Aksenov, and S. Polikhov, "Detonation initiation by controlled triggering of electric discharges," Journal of Propulsion and Power, vol. 19, no. 4, pp. 573-580, 2003. DOI: https://doi. org/10.2514/2.6168.

[18] J. A. Gray and D. A. Lacoste, "Effects of nanosecond repetitively pulsed plasma discharges on a propagating hydrogen-air flame," in AIAA Scitech 2019 Forum, 2019, p. 0750. DOI: https ://doi.org/10.2514/6.20190750 .

[19] G. Ciccarelli, C. Johansen, and M. Hickey, "Flame acceleration enhancement by distributed ignition points," Journal of propulsion and power, 
vol. 21, no. 6, pp. 1029-1034, 2005. DOI: https://doi.org/10.1016/ j.pecs.2007.11.002.

[20] G. E. Box, W. G. Hunter, J. S. Hunter, and al., Statistics for experimenters. John Wiley and sons New York, 1978, vol. 664.

[21] D. M. Valiev, V. Akkerman, M. Kuznetsov, L.-E. Eriksson, C. K. Law, and V. Bychkov, "Influence of gas compression on flame acceleration in the early stage of burning in tubes," Combustion and Flame, vol. 160, no. 1, pp. 97-111, 2013. DOI: https ://doi .org/10.1016/j . combustflame. 2012.09.002.

[22] X. Yang, M. Yu, K. Zheng, P. Luan, and S. Han, "On the propagation dynamics of lean h2/co/air premixed flame," International Journal of Hydrogen Energy, vol. 45, no. 11, pp. 7210-7222, 2020. DOI: https : //doi.org/10.1016/j.ijhydene.2019.12.116.

[23] C. Clanet and G. Searby, "On the "tulip flame" phenomenon," Combustion and Flame, vol. 105, no. 1-2, pp. 225-238, 1996. DOI: https: //doi.org/10.1016/0010-2180(95)00195-6.

[24] V. Bychkov, V. Akkerman, G. Fru, A. Petchenko, and L.-E. Eriksson, "Flame acceleration in the early stages of burning in tubes," Combustion and Flame, vol. 150, no. 4, pp. 263-276, 2007. DOI: https://doi.org/ 10.1016/j. combustflame.2007.01.004.

[25] J. Kurylo, H. Dwyer, and A. Oppenheim, "Numerical analysis of flowfields generated by accelerating flames," AIAA Journal, vol. 18, no. 3, pp. 302-308, 1980. DOI: https://doi.org/10.2514/3.50763.

[26] B. Ponizy, A. Claverie, and B. Veyssière, "Tulip flame-the mechanism of flame front inversion," Combustion and Flame, vol. 161, no. 12, pp. 30513062, 2014. DOI: https://doi .org/10.1016/j . combustflame. 2014. 06.001.

[27] H. Xiao, R. W. Houim, and E. S. Oran, "Formation and evolution of distorted tulip flames," Combustion and Flame, vol. 162, no. 11, pp. 40844101, 2015. DOI: https://doi .org/10.1016/j . combustflame. 2015. 08.020 .

[28] V. Akkerman, V. Bychkov, A. Petchenko, and L.-E. Eriksson, "Flame oscillations in tubes with nonslip at the walls," Combustion and Flame, vol. 145, no. 4, pp. 675-687, 2006. DOI: https://doi.org/10.1016/j . combustflame.2006.01.013.

[29] L. R. Boeck, P. Katzy, J. Hasslberger, A. Kink, and T. Sattelmayer, "The gravent DDT database," Shock Waves, vol. 26, no. 5, pp. 683-685, 2016. DOI: https://doi.org/10.1007/s00193-016-0629-0.

[30] H. Xiao, Q. Wang, X. Shen, W. An, Q. Duan, and J. Sun, "An experimental study of premixed hydrogen/air flame propagation in a partially open duct," International Journal of Hydrogen Energy, vol. 39, no. 11, pp. 6233-6241, 2014. DOI: https ://doi .org/10.1016/j . ijhydene. 2013.05 .003$.

[31] H. Guenoche, "Flame propagation in tubes and in closed vessels," in AGARDograph, vol. 75, Elsevier, 1964, pp. 107-181. DOI: https://doi. org/10 . 1016/B978-1-4831-9659-6.50008-1. 
[32] J.-C. Leyer and N. Manson, "Development of vibratory flame propagation in short closed tubes and vessels," in International Symposium on Combustion, Elsevier, vol. 13, 1971, pp. 551-558. DOI: https://doi. org/10.1016/S0082-0784(71)80056-5.

[33] S. Kerampran, D. Desbordes, and B. Veyssiere, "Study of the mechanisms of flame acceleration in a tube of constant cross section," Combustion Science and Technology, vol. 158, no. 1, pp. 71-91, 2000. DOI: https://doi.org/10.1080/00102200008947328.

[34] S. Kerampran, D. Desbordes, B. Veyssière, and L. Bauwens, "Flame propagation in a tube from closed to open end," in 39th Aerospace Sciences Meeting and Exhibit, 2001, p. 1082. DOI: https://doi.org/10. 2514/6.2001-1082.

[35] J. Gray, J. P. Moeck, and C. O. Paschereit, "Flame propagation from the closed end of an open-ended tube: An analysis of the effects of fuel type, tube length, and equivalence ratio and an insight into flame dynamics," in 49th AIAA/ASME/SAE/ASEE Joint PropulsionConference, 2013, p. 3656. DOI: https://doi.org/10.2514/6.2013-3656.

[36] F. F. Fachini and L. Bauwens, "Oscillatory flame propagation: Coupling with the acoustic field," Proceedings of the Combustion Institute, vol. 34, no. 2, pp. 2043-2048, 2013. DOI: https://doi.org/10.1016/j.proci. 2012.05 .032$.

[37] S. Qi, Y. Du, P. Zhang, G. Li, S. Wang, Y. Li, and T. Dong, "Experimental study of gasoline vapor deflagration in a duct with an open end," Combustion and Flame, vol. 193, pp. 16-24, 2018. DOI: https : //doi.org/10.1016/j.combustflame.2018.02.022.

[38] M. Kuznetsov, A. Lelyakin, V. Alekseev, and I. Matsukov, "Detonation transition in relatively short tubes," in 30th International Symposium on Shock Waves 1, Springer, 2017, pp. 481-485. DOI: https ://doi . org/10.1007/978-3-319-46213-4_83.

[39] A. Khokhlov, E. Oran, and G. Thomas, "Numerical simulation of deflagrationto-detonation transition: The role of shock-flame interactions in turbulent flames," Combustion and Flame, vol. 117, no. 1-2, pp. 323-339, 1999. DOI: https://doi.org/10.1016/S0010-2180(98)00076-5.

[40] G. Thomas, R. Bambrey, and C. Brown, "Experimental observations of flame acceleration and transition to detonation following shock-flame interaction," Combustion Theory and Modelling, vol. 5, no. 4, pp. 573594, 2001. DOI: https://doi.org/10.1088/1364-7830/5/4/304.

[41] J. Yanez and M. Kuznetsov, "Experimental study and theoretical analysis of a 'strange wave'," Combustion and Flame, vol. 167, pp. 494-496, 2016. DOI: https://doi.org/10.1016/j . combustflame. 2016.02.004. 
Appendix

\section{Response Surface Methods - mathematical framework}

A Response Surface Model including potential interactions among $n$ parameters reads:

$$
\underset{\left(2^{n}, 1\right)}{\boldsymbol{y}}=\underset{\left(2^{n}, 2^{n}\right)\left(2^{n}, 1\right)}{\boldsymbol{a}}
$$

which defines a $2^{n} \times 2^{n}$ system of linear equations, where $\boldsymbol{y}$ is the vector of responses (i.e. experimental output), $\boldsymbol{X}$ is a Hadamard matrix ${ }^{1}$ defining the test conditions from a full experimental matrix, and $\boldsymbol{a}$ is the vector of coefficients sought for. More specifically, the magnitude of the elements of vector $\boldsymbol{a}$ allow to evaluate the influence of a particular parameter, $a_{1}$ for parameter 1 , or the interactions between different parameters, $a_{12}$ for the interaction of parameter 1 with parameter 2 , and so on, on the response, $\boldsymbol{y}$. Accounting for $n=4$ parameters results in 16 coefficients that need being determined (see Eq. 6) which entails performing 16 tests at different conditions, $\boldsymbol{X}$, to obtain an equal number of responses, $\boldsymbol{y}$. Instead of directly computing these coefficients to construct a response surface, the model can be simplified by neglecting high order interactions, among three or more parameters for example (which are less likely) and by using aliases, $\boldsymbol{l}$, in which the different experimental factors are confounded based on the experimental matrix reduction methodology described in [14].

Doing so, the following reduced model is obtained:

$$
\underset{(8,1)}{\boldsymbol{y}}=\underset{(8,8)(8,1)}{\boldsymbol{X}_{\boldsymbol{s}}} \underset{\boldsymbol{l}}{\boldsymbol{l}}
$$

where the elements of $\boldsymbol{l}$ are determined by combining the elements of $\boldsymbol{a}$ as follows:

$$
\begin{gathered}
l_{0} \approx a_{0} ; \quad l_{1} \approx a_{1}+a_{24} ; \quad l_{2} \approx a_{2}+a_{14} ; \quad l_{3} \approx a_{3} ; \quad l_{4} \approx a_{4}+a_{12} \\
l_{23} \approx a_{23} ; \quad l_{13} \approx a_{13} ; \quad l_{34} \approx a_{34}
\end{gathered}
$$

subscripts 1,2,3 and 4 correspond to the settling time, ignition offset, material and ignition energy, respectively; the remaining subscripts are interactions among parameters. As per Eq. 7 only eight tests are needed to determine the aliases vector, $\boldsymbol{l}$. The conditions at which the tests need being carried out are given in Table 3, constructed from the Hadamard matrix, $\boldsymbol{X}_{\boldsymbol{s}}$, by arbitrarily assigning values of +1 or -1 to the limits of each parameter, i.e., -1 for small ignition offset, glass, $E_{\min }$ and $t_{\min }$ and +1 for large ignition

\footnotetext{
1 A square matrix whose entries are either +1 or -1 and whose rows are mutually orthogonal; used by statisticians to estimate the variance of a parameter estimator.
} 
offset, plastic, $E_{\max }$ and $t_{\max }$. The remaining four columns (5, 6, 7 and 8$)$ that would complete Table 3 are the product of ignition offset and material; settling time and material; ignition energy and material; and settling time, ignition offset, and ignition energy. Finally, using the mean front position, $\bar{x}$, as our response, $\boldsymbol{y}$, we can solve for $\boldsymbol{l}$ and plot their temporal evolution. The results are shown in Fig. 8 of section 3.3.

Table 3 Experimental conditions determined following [14] to compute the alias vector $\boldsymbol{l}$; all tests were performed using Igniter 1 as an ignition source.

\begin{tabular}{ccccc}
\hline Test Number & Settling time & Ignition offset & Material & Ignition energy \\
\hline 1 & $\min$ & small & glass & $\max$ \\
2 & $\max$ & small & glass & $\min$ \\
3 & $\min$ & large & glass & $\min$ \\
4 & $\max$ & large & glass & $\max$ \\
5 & $\min$ & small & plastic & $\max$ \\
6 & $\max$ & small & plastic & $\min$ \\
7 & $\min$ & large & plastic & $\min$ \\
8 & $\max$ & large & plastic & $\max$ \\
\hline
\end{tabular}

\section{Additional parameters tested}

\subsection{Settling time between end of filling and ignition}

Figure 16 shows front position results comparing two settling times, $t_{\min }=15 \mathrm{~s}$ and $t_{\max }=60 \mathrm{~s}$ for glass (top) and polycarbonate (bottom).

With glass, $d_{t_{\min }}$ and $d_{t_{\max }}<5 \mathrm{~mm}$, and subsequently increases to $\sim 30$ $\mathrm{mm}$ for $t \geq 5 \mathrm{~ms}$; furthermore, all three deviations $\left(d_{t_{\min }}, d_{t_{\max }}\right.$ and $\left.d_{\mathrm{g}}\right)$ follow the same evolution, with differences of less than $5 \mathrm{~mm}$. With plastic both deviations are similar $(\sim 10 \mathrm{~mm})$ for $t \leq 5 \mathrm{~ms}$, and gradually increase to $\sim 20 \mathrm{~mm}$. For $5 \mathrm{~ms}<t<11 \mathrm{~ms}$, the maximum difference $\left|d_{g}-d_{t_{\max }}\right|_{\max }$ reaches $15 \mathrm{~mm}$; the flame positions follow different paths before rejoining near the end. Although $d_{g}$ is greater than both $d_{t_{\max }}$ and $d_{t_{\max }}$ in this time interval, the difference is small compared to the usual scattering of the results. Besides, all three deviations $\left(d_{t_{\min }}, d_{t_{\max }}\right.$ and $\left.d_{\mathrm{g}}\right)$ are equal close to the end $(\sim 20 \mathrm{~mm})$; $d_{g}$ is larger for $t<11 \mathrm{~ms}$ which could imply an interaction between two parameters, namely the settling time and material, as this is observed with this material only. However, since $\left|d_{\mathrm{g}}-d_{t_{\max }}\right|_{\max } \leq 5 \mathrm{~mm}$ with glass, this suggests that the settling time is not responsible for the discrepancies shown. Finally, the coefficient $l_{13}$ of the response model, shown in section 3.3, rules out the potential interaction between these parameters, as its value is low compared to $l_{2}$. Due to size of the channel used, settling times of at least $15 \mathrm{~s}$ are sufficient to avoid potential effects on FA. 


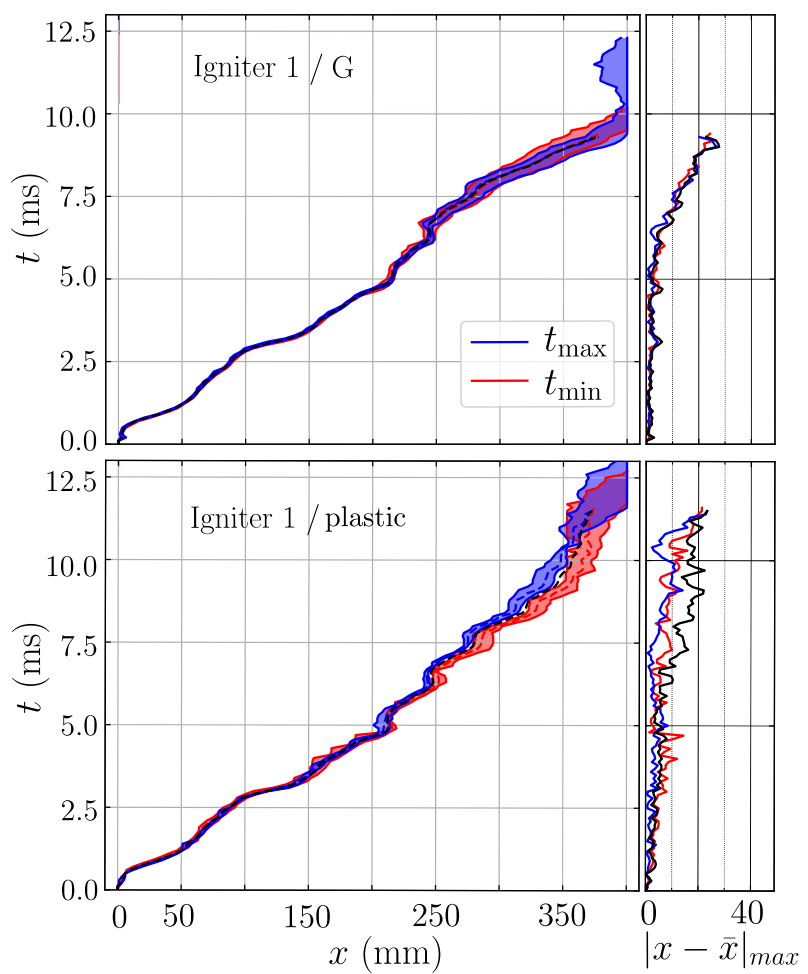

Fig. 16 Influence of settling time before ignition of mixture on mean flame position, $\bar{x}$ (dashed line). Absolute maximum deviation from mean value $|x-\bar{x}|_{\max }$. Top: glass (sample size $=6$ tests), Bottom: plastic (sample size $=6$ tests). Configuration: small ignition offset and Igniter 1; conditions: stoichiometric $\mathrm{H}_{2}$-air at $p_{0}=100 \mathrm{kPa}$, and $T_{0}=290 \mathrm{~K}$; settling times: $t_{\max }=60 \mathrm{~s}, t_{\min }=15 \mathrm{~s}$.

\subsection{Material utilized for optical access}

In Fig. 17 we compare the front position data changing the material used for optical access for small ignition offset (top) and large ignition offset (bottom).

For small ignition offset, deviations are small up to $t \sim 5 \mathrm{~ms}$ for both materials. Thereafter, $d_{\text {plastic }}$ increases to $20 \mathrm{~mm}$ whereas $d_{\text {glass }}$ stays below $30 \mathrm{~mm}$. The global deviation, $d_{\mathrm{g}}$, follows that of plastic closely, once again showing that the materials are not responsible for the observed scatter. Tests performed with large ignition offset confirm this trend. For large ignition offset, $d_{\text {plastic }}<10 \mathrm{~mm}$ throughout the propagation period; $d_{\text {glass }}<10 \mathrm{~mm}$ for $t \leq 5 \mathrm{~ms}$ increasing up to a maximum of $25 \mathrm{~mm}$. Note that since both materials have similar roughness [16] this was somewhat expected, however, the main purpose of evaluating this parameter was to assess its effect on the chemiluminescence signal; the same luminous intensity was observed, suggesting that plastic does not burn during flame propagation and acceleration. Continuous testing, on the other hand, does leave visible marks on the plastic but, based 


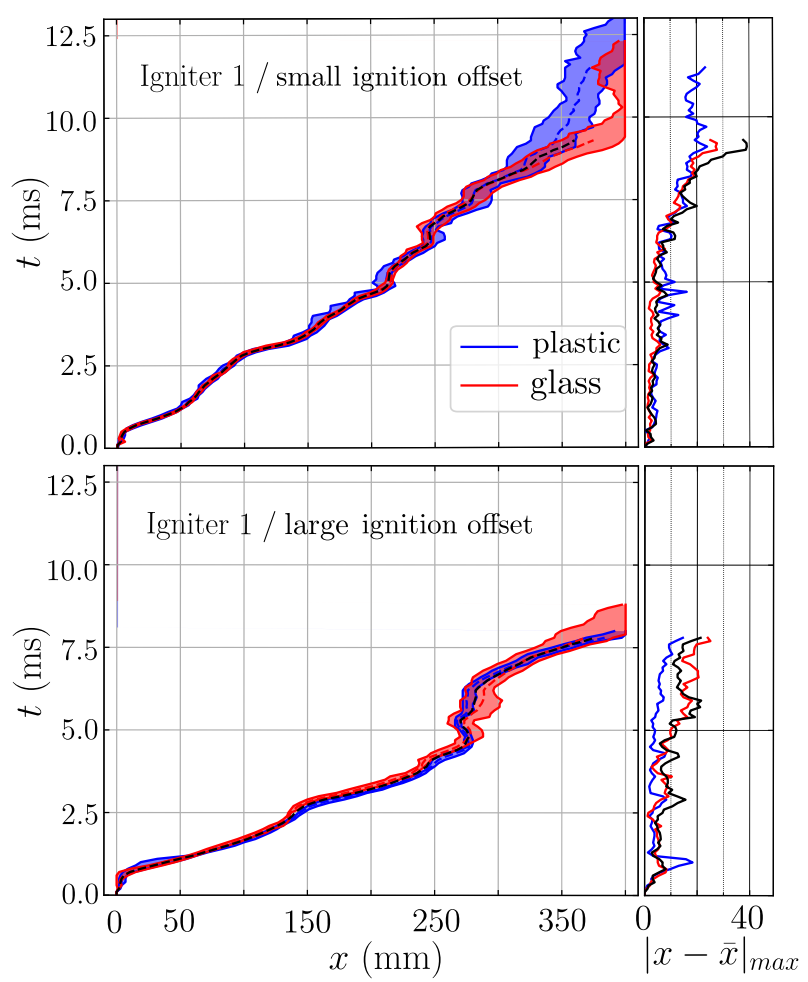

Fig. 17 Influence of window material on mean flame position, $\bar{x}$ (dashed line). Absolute maximum deviation from mean value $|x-\bar{x}|_{\max }$. Top: small ignition offset (sample size $=$ 12 tests), Bottom: large ignition offset (sample size $=10$ tests). Configuration: Igniter 1 for both figures; conditions: stoichiometric $\mathrm{H}_{2}$-air at $p_{0}=100 \mathrm{kPa}$, and $T_{0}=290 \mathrm{~K}$.

on our data, these do not seem to perturb the recorded flame propagation. The global deviation remains above their intrinsic counterpart until $t \sim 8 \mathrm{~ms}$ slightly decreasing before the open end of the channel. The time of arrival of the flame with glass being smaller than with plastic, a bifurcation is visible. This discrepancy was not seen with large ignition offset, where in contrast, glass presents a larger deviation. This result may again imply an interaction between two parameters, namely ignition offset and materials, an outcome similar to that described in Fig. 16. It is not straightforward to conclusively determine why this is the case however, a lead would be the different stiffness of plastic and the aluminum frame holding the glass, resulting in different acoustic impedances in a section prone to acoustic effects (the open end of the channel). In summary, using glass or plastic did not show significant deviations until the open end was approached. 


\section{Effect of ignition offset for less diluted mixtures}

As the nitrogen dilution is reduced, the sensitivity to detonation onset increases, 20 additional experiments were conducted to determine whether the behaviors observed for $\mathrm{H}_{2}$-air at small/large ignition offsets also apply to stoichiometric $\mathrm{H}_{2}-\mathrm{O}_{2}$ mixtures. To choose appropriate framing rates to capture detonations the steady Chapman-Jouguet velocity is used $(2,840 \mathrm{~m} / \mathrm{s}) ; 360,000$ fps, the maximum allowed by the camera with the spatial resolution specified in section 2.2, are thus sufficient. A significantly shorter exposure time (159 ns) is necessary to avoid saturation during DDT and detonation propagation. Note however that this choice decreases the accuracy of the luminous front detection during the flame acceleration stage. Figure 18 shows the $x-t$ diagram. DDT was not systematically captured as in most cases (18 out of 20 tests) detonation onset occurred outside of the viewing window (i.e. $x \geq 400 \mathrm{~mm}$ ).

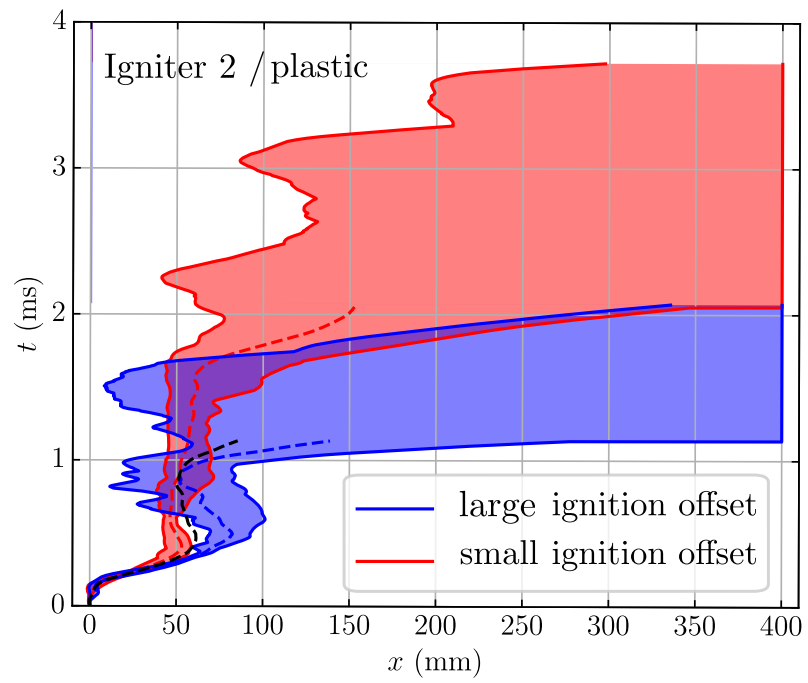

Fig. 18 Influence of ignition offset on mean flame position $\bar{x}$ (dashed line). Blue: large ignition offset (sample size $=7$ tests), Red: small ignition offset (sample size $=13$ tests). Configuration: Igniter 2 and plastic; condition: stoichiometric $\mathrm{H}_{2}-\mathrm{O}_{2}$ at $p_{0}=100 \mathrm{kPa}$, and $T_{0}=290 \mathrm{~K}$

For the remaining two cases, one with a small ignition offset and one with a large ignition offset, DDT occured at $x=365 \mathrm{~mm}$ and $x=377 \mathrm{~mm}$, respectively, showing that the ignition offset does not seem to influence significantly the run-up distances prior to DDT compared to the detonation onset time, $t=2.43 \mathrm{~ms}$ for small ignition offset, and $t=1.23 \mathrm{~ms}$ for large ignition offset. Note that the backward motion shown in our data could in principle come from the reflection of precursor shocks as expansions from the open end of the channel, and their subsequent interaction with the reactive front as previously discussed in [38], however, this may not be the case here. The camera settings 
required to capture the detonation phase accurately (159 ns exposure time) do not perform well during the FA phase resulting in incorrect estimates of the front position; this represents one of the main limitations of direct observation to study DDT in hydrogen mixtures compared to hydrocarbons [22]. The steep acceleration phase shown in the figure is nonetheless correctly detected, but does not correspond to actual transition to detonation as computed velocities are in the range of $u_{f}=800-1800 \mathrm{~m} / \mathrm{s}$. Only for the aforementioned two cases where DDT occurred within the viewing window velocities reached $2800 \mathrm{~m} / \mathrm{s}$. Abrupt acceleration leading to quasi-steady propagation velocities have been identified previously to have complex structures that include planar shocks trailed by funnel shaped flames [39]-[41], unfortunately with direct observation we cannot verify if this is the case for our data. Finally, it is worth mentioning that pollution due to the presence of sodium in air, does not seem to contribute to enhanced light emissions in the combustion products as we observed the same intensity levels in the raw images captured with $\mathrm{H}_{2}-\mathrm{O}_{2}$ (not shown). 